\title{
Dosimetric and Efficiency Comparisons between Volumetric Modulated Arc Therapy and IMRT for Cervical-Thoracic Esophageal Cancers
}

\author{
Yuxin Duan ${ }^{*}$, Xi Liu' ${ }^{*}$, Zhongqiang Liu ${ }^{3 *}$, Ning Zhan", Zongda Zhu4, Zhenxiang Deng4\# \\ ${ }^{1}$ Department of Radiation Oncology, Southern Theater Command Air Force Hospital, Guangzhou, China \\ ${ }^{2}$ School of Medical Information and Engineering, Guangdong Pharmaceutical University, Guangzhou, China \\ ${ }^{3}$ Department of Radiation Oncology, Nanfang Hospital, Southern Medical University, Guangzhou, China \\ ${ }^{4}$ Department of Radiation Oncology, Xiang'an Hospital of Xiamen University, School of Medicine, Xiamen University, \\ Xiamen, China \\ Email: "ghost_fx135@163.com
}

How to cite this paper: Duan, Y.X., Liu, X., Liu, Z.Q., Zhan, N., Zhu, Z.D. and Deng, Z.X. (2021) Dosimetric and Efficiency Comparisons between Volumetric Modulated Arc Therapy and IMRT for Cervical-Thoracic Esophageal Cancers. Journal of Cancer Therapy, 12, 593-601.

https://doi.org/10.4236/jct.2021.1211051

Received: October 2, 2021

Accepted: October 30, 2021

Published: November 2, 2021

Copyright (c) 2021 by author(s) and Scientific Research Publishing Inc. This work is licensed under the Creative Commons Attribution International License (CC BY 4.0).

http://creativecommons.org/licenses/by/4.0/

\section{(c) (i) Open Access}

\begin{abstract}
Purpose: This study aims to evaluate the treatment plans of Volumetric-modulated arc therapy (VMAT) and intensity-modulated radiation therapy (IMRT) techniques for cervical-thoracic esophageal cancers. Methods and Materials: Sixty patients were retrospectively identified. Several parameters were evaluated based on target conformity and dose-volume histograms of organs at risk (lung, spinal cord, and heart). A phantom for time comparison was also assessed for each plan. Results: The IMRT plans (5f-IMRT: V95\% $=99.4 \pm 0.3$, 7f-IMRT: V95\% $=99.8 \pm 0.1)$ results in better PTV coverage than RA plans (Single-arc: V95\% $=95.8 \pm 3.2$, Double-arc: V95\% $=$ $95.4 \pm 2.3)$. The target dose conformity of the 5 f-IMRT plan was inferior to all plans $(\mathrm{CI}=70.4 \pm 7.1)$. The Single-arc plan achieved the best conformity $(\mathrm{CI}=72.5 \pm 4.6)$, whereas the Double-arc plan $(\mathrm{CI}=72.1 \pm 5.1)$ was slightly inferior to the Single-arc plan but superior to the 7f-IMRT plan (CI $=71.7 \pm$ 8.6). The total MU was reduced by $42.1 \%$ in VMAT plan. The average MU needed to deliver the dose of $60 \mathrm{~Gy}$ for Single-arc $(423.5 \pm 52.1 \mathrm{MU})$ was found to be the least. Similarly, the average MU for the 5f-IMRT, 7f-IMRT and Double-arc were 868.2 $\pm 182.0 \mathrm{MU}, 870.0 \pm 225.3 \mathrm{MU}$ and $548.8 \pm 47.2$ MU, respectively. The delivery time in VMAT plans was reduced from 193.8 seconds to 99.2 seconds by around $48.8 \%$ compared to IMRT plans.
\end{abstract}

*Authors contribute equally.

${ }^{\#}$ Corresponding author. 
Conclusion: For similar PTV parameters, VMAT delivers a lower dose to organs at risk than IMRT in a shorter time, and this has warranted clinical implementation.

\section{Keywords}

Esophageal, Dosimetric, IMRT, VMAT

\section{Introduction}

Chemo-radiation has emerged as a standard method in the treatment of cervical-thoracic esophageal cancer [1] [2]. However, because of complexity of anatomic structures in head and neck, especially in cervical-thoracic esophagus, radiotherapy presents a particularly difficult treatment planning problem [3].

In recent years, volumetric-modulated arc therapy (VMAT) has become more and more important in the treatment of several tumor types and has been shown to provide significant dosimetric and delivery benefits over intensity-modulated radiation therapy (IMRT) [4]-[9]. The term intensity-modulated radiation therapy (IMRT) refers to a radiation therapy technique in which a nonuniform fluence is delivered to the patient from any given position of the treatment beam to optimize the composite dose distribution. The delivery of a rotational cone beam with variable shape and intensity is commonly called volumetric-modulated arc therapy (VMAT). In a VMAT treatment, the gantry moves continuously, with the MLC leaves and dose rate varying throughout the arc. The TPS computes the dose by sampling the delivery at a number of discrete gantry angles [10]. So far, there have been no published reports on the advantages of VMAT use in cervical-thoracic esophageal patients compared with IMRT. Volumetric arc modulation using VMAT enables IMRT-like dose distributions to be delivered using a single and/or multiple rotations of the gantry. Recent studies report the efficacy of RapidArc (RA) over conventional IMRT in terms of PTV conformity and reduction in organs at risk (OAR) doses. These studies have further demonstrated the ability of VMAT to reduce the number of monitor units and overall treatment times when compared with IMRT. The purpose of this study is to investigate whether VMAT has the capability to deliver plans that are superior to IMRT for cervical-thoracic esophageal cancers [11] [12] [13].

\section{Methods and Materials}

Sixty patients with pathologically proven primary and secondary cervical-thoracic esophageal squamous carcinoma were selected for the study, the median age was 65. Patients were immobilized in supine position using a thermoplastic mask system with active fixation of light points and scanned with a Philips Brilliant spiral CT (Philips Brilliant, Cleveland, $\mathrm{OH}$ ) according to standard procedures with $3 \mathrm{~mm}$ slice spacing. 


\section{Target Contour and Planning}

The clinical target volume (CTV) included the esophageal tumor, with a margin for microscopic tumor extension, and the adjacent lymph nodes. For the planning tumor volume (PTV), a three-dimensional margin of $15 \mathrm{~mm}$ was added to the CTV to account for the variability in patient setup, uncertainty in target definition, and organ motion. The following OARs were contoured: lungs, heart, and spinal cord. The healthy tissue was defined as the patient's volume covered by the body volume excluding the PTV.

The goal of treatment planning was to get a good coverage of PTV while sparing normal tissues. All plans were normalized to $60 \mathrm{~Gy}$ at 30 fractions at $6 \mathrm{MV}$ for all the patients plans. Rapaid and IMRT plans were generated for each patient using Varian Eclipse treatment planning system (Clinical version 15.6.01, Varian USA). All plans were optimized to reach clinically acceptable PTV coverage and organ at risk (OAR) sparing. At least $95 \%$ of the PTV must be covered by $95 \%$ of the prescription dose.

\section{Evaluation}

Quantitative evaluation of plans was performed by means of standard dose volume histogram (DVH). To evaluate PTV coverage, the values of D98\% and D21\% (dose received by the $98 \%$ and $2 \%$ of the volume) were defined as metrics for minimum and maximum doses and consequently reported. V95\% (the volume receiving at least $95 \%$ of the prescribed dose) was eported as the target coverage. The degree of conformity of the plans was measured with the Conformity Index (CI), the CI was defined as the ratio between the patient volume receiving at least $95 \%$ of the prescribed dose and the volume of the PTV. For OARs and health tissues, the analysis included the mean dose and a set of appropriate VX and DY values. To evaluate the difference in treatment time between VMAT and IMRT, total MU and the delivery time were also accounted based on known machine parameters. To determine statistical significance, the paired, two-tailed Student's t-test and the paired, two-tailed Wilcoxon's signed ranks test were used. $\mathrm{p}$-value $\mathrm{p}<0.05$ were considered to be significant.

\section{Result}

The dose distributions for the four plans were shown for one patient in Figure 1. In Tables 1-5, an overview of the numerical findings from average DVH analysis on OARs and PTV are reported as mean values \pm standard deviation to assess for the relative inter-patient variability. The significant differences between delivery methods for OARs and PTV are also reported in the table.

\section{PTV Dosimetric Parameters}

Clinically acceptable plans of VMAT and IMRT were completed by all the sixty patients. The IMRT plans (5f-IMRT: $\mathrm{V}_{95 \%}=99.4 \pm 0.3$, 7f-IMRT: $\mathrm{V}_{95 \%}=$ $99.8 \pm 0.1$ ) results in better PTV coverage than RA plans (Single-arc: $\mathrm{V}_{95 \%}=95.8$ \pm 3.2 , Double-arc: $\mathrm{V}_{95 \%}=95.4 \pm 2.3$ ). The target dose conformity of the 5f-IMRT plan was inferior to all plans $(\mathrm{CI}=70.4 \pm 7.1)$. The Single-arc plan achieved the 
Table 1. Summary of numeric analysis from DVH for target volumes.

\begin{tabular}{cccccc}
\hline Parameter & 5f-IMRT & 7f-IMRT & RA_SA & RA_DA & p \\
\hline $\begin{array}{c}\text { Conformity } \\
\text { Index }\end{array}$ & $0.704 \pm 0.071$ & $0.717 \pm 0.086$ & $0.72 .5 \pm 0.046$ & $0.72 .1 \pm 0.051$ & NS \\
PTV & & & & & \\
$\left(656 \pm 112 \mathrm{~cm}^{3}\right)$ & & & & \\
$\mathrm{D}_{2 \%}(\mathrm{~Gy})$ & $64.0 \pm 1.8$ & $63.6 \pm 1.5$ & $63.5 \pm 1.1$ & $61.8 \pm 0.9$ & c, e, f \\
$\mathrm{D}_{98 \%}(\mathrm{~Gy})$ & $58.0 \pm 0.6$ & $58.6 \pm 0.8$ & $56.6 \pm 0.8$ & $56.3 \pm 0.6$ & b, c, d, e \\
$\mathrm{V}_{95 \%}(\%)$ & $99.4 \pm 0.3$ & $99.8 \pm 0.1$ & $95.8 \pm 3.2$ & $95.4 \pm 2.3$ & a, b, c, d, e \\
\hline
\end{tabular}

Statistically significant differences $(\mathrm{p}<0.05)$ of paired t-test analysis for four plans; a: 5f-IMRT vs. 7f-IMRT; b: 5f-IMRT vs. RA_SA; c: 5f-IMRT vs. RA_DA; d: 7f-IMRT vs. RA_SA; e: 7f-IMRT vs. RA_DA; f: RA_SA vs. RA_DA.

Table 2. Maximum of spinal cord recorded from the DVHs.

\begin{tabular}{cccccc}
\hline Parameter & 5f-IMRT & 7f-IMRT & RA_SA & RA_DA & p \\
\hline $\mathrm{D}_{\max }(\mathrm{Gy})$ & $42.3 \pm 1.2$ & $41.4 \pm 2.4$ & $42.9 \pm 1.7$ & $40.7 \pm 1.8$ & NS \\
\hline
\end{tabular}

Statistically significant differences $(\mathrm{p}<0.05)$ of paired t-test analysis for four plans; a: 5f-IMRT vs.7f-IMRT; b: 5f-IMRT vs. RA_SA; c: 5f-IMRT vs. RA_DA; d: 7f-IMRT vs. RA_SA; e: 7f-IMRT vs. RA_DA; f: RA_SA vs. RA_DA. NS: Not significant.

Table 3. Lung dose calculated from the DVHs.

\begin{tabular}{cccccc}
\hline Parameter & 5f-IMRT & 7f-IMRT & RA_SA & RA_DA & p \\
\hline $\mathrm{D}_{\text {mean }}(\mathrm{Gy})$ & $15.0 \pm 2.1$ & $15.1 \pm 2.0$ & $14.5 \pm 2.3$ & $14.2 \pm 2.0$ & b, c, d, e \\
$\mathrm{V}_{5 \text { Gy }}(\%)$ & $60.2 \pm 13.3$ & $60.5 \pm 12.9$ & $60.2 \pm 13.9$ & $60.9 \pm 14.0$ & NS \\
$\mathrm{V}_{10 \text { Gy }}(\%)$ & $50.5 \pm 9.0$ & $52.8 \pm 10.3$ & $52.4 \pm 12.0$ & $52.5 \pm 11.5$ & $\mathrm{a}$ \\
$\mathrm{V}_{20 \text { Gy }}(\%)$ & $30.2 \pm 2.5$ & $30.3 \pm 1.8$ & $28.4 \pm 2.7$ & $25.8 \pm 1.6$ & b, c, e, f \\
$\mathrm{V}_{\text {30 Gy }}(\%)$ & $16.9 \pm 1.6$ & $16.1 \pm 1.8$ & $15.3 \pm 1.3$ & $14.6 \pm 1.6$ & a, b, c, d, e \\
$\mathrm{V}_{40 \text { Gy }}(\%)$ & $9.5 \pm 2.0$ & $9.5 \pm 1.8$ & $8.8 \pm 1.2$ & $9.2 \pm 1.6$ & NS \\
$\mathrm{V}_{50 \text { Gy }}(\%)$ & $5.1 \pm 1.4$ & $5.0 \pm 1.4$ & $4.2 \pm 1.0$ & $4.8 \pm 1.1$ & b, d \\
\hline
\end{tabular}

Statistically significant differences $(\mathrm{p}<0.05)$ of paired t-test analysis for four plans; a: 5f-IMRT vs. 7f-IMRT; b: 5f-IMRT vs. RA_SA; c: 5f-IMRT vs. RA_DA; d: 7f-IMRT vs. RA_SA; e: 7f-IMRT vs. RA_DA; f: RA_SA vs. RA_DA. NS: Not significant.

Table 4. Heart dose calculated from the DVHs.

\begin{tabular}{cccccc}
\hline Parameter & 5f-IMRT & 7f-IMRT & RA_SA & RA_DA & p \\
\hline Mean (Gy) & $10.4 \pm 9.5$ & $10.4 \pm 9.6$ & $10.1 \pm 9.3$ & $10.1 \pm 9.0$ & d \\
$\mathrm{D}_{35 \%}$ (Gy) & $9.0 \pm 12.8$ & $9.0 \pm 12.9$ & $8.6 \pm 11.2$ & $8.4 \pm 11.0$ & NS \\
\hline
\end{tabular}

Statistically significant differences $(p<0.05)$ of two-paired Wilcoxon's signed ranks test for four plans; a: 5f-IMRT vs. 7f-IMRT; b: 5f-IMRT vs. RA_SA; c: 5f-IMRT vs. RA_DA; d: 7f-IMRT vs. RA_SA; e: 7f-IMRT vs. RA_DA; f: RA_SA vs. RA_DA. NS: Not significant. 


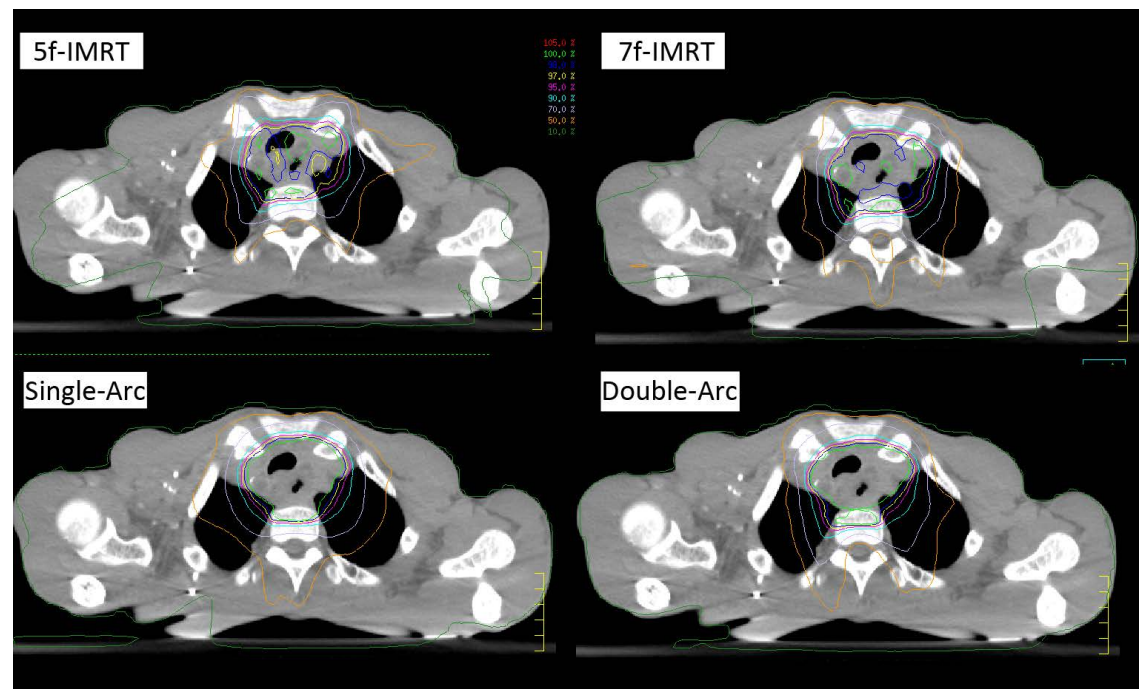

Figure 1. Isodose distributions of 5f-IMRT, 7f-IMRT, single-arc RA, and double-arc RA plans of one case.

Table 5. Total MU and delivery time.

\begin{tabular}{cccccc}
\hline Parameter & 5f-IMRT & 7f-IMRT & RA_SA & RA_DA & p \\
\hline Total MU & $868.2 \pm 182.0$ & $870.0 \pm 225.3$ & $423.5 \pm 52.1$ & $548.8 \pm 47.2$ & b, c, d, e, f \\
Delivery Time (s) & $198.7 \pm 36.5$ & $198.0 \pm 44.9$ & $84.7 \pm 10.7$ & $113.7 \pm 9.4$ & b, c, d, e, f \\
\hline
\end{tabular}

Statistically significant differences $(\mathrm{p}<0.05)$ of paired $\mathrm{t}$-test analysis for four plans; a: 5f-IMRT vs. 7f-IMRT; b: 5f-IMRT vs. RA_SA; c: 5f-IMRT vs. RA_DA; d: 7f-IMRT vs. RA_SA; e: 7f-IMRT vs. RA_DA; f: RA_SA vs. RA_DA. NS: Not significant.

best conformity $(\mathrm{CI}=72.5 \pm 4.6)$, whereas the Double-arc plan $(\mathrm{CI}=72.1 \pm 5.1)$ was slightly inferior to the Single-arc plan but superior to the 7f-IMRT plan (CI $=71.7 \pm 8.6)$. But there were no statistically significant Differences between the plans.

\section{Spinal Cord}

All plans complied with the planning objective of $45 \mathrm{~Gy}$ as maximum dose to the spinal cord. The double-arc plan spared dose most of all the plans. But no statistically significant differences were observed between the plans.

\section{Lung}

As summarized in Table 3, MLD, lung $\mathrm{V}_{20}$ and $\mathrm{V}_{30}$ in VMAT plans were lower than in IMRT plans $(\mathrm{p}<0.05) . \mathrm{V}_{20}$ reduced from $30.2 \% \pm 2.5 \%$ in 5 f-IMRT and $30.3 \% \pm 1.8 \%$ in 7 f-IMRT to $28.4 \% \pm 2.7 \%$ in Single-arc and $25.8 \% \pm 1.6 \%$ in Double-arc. $\mathrm{V}_{30}$ reduced from $16.9 \% \pm 1.6 \%$ in 5 f-IMRT and16.1\% $\pm 1.8 \%$ in 7f-IMRT to $15.3 \% \pm 1.3 \%$ in Single-arc and $14.6 \% \pm 1.6 \%$ in Double-arc. MLD reduced from $15.0 \pm 2.1 \mathrm{~Gy}$ in 5f-IMRT and15.1 $\pm 2.0 \mathrm{~Gy}$ in 7f-IMRT to $14.5 \pm$ 2.3 Gy in Single-arc and 14.2 $\pm 2.0 \mathrm{~Gy}$ in Double-arc. $\mathrm{V}_{5}, \mathrm{~V}_{10}$ and $\mathrm{V}_{40}$ showed no difference between IMRT and VMAT plans. Lung $\mathrm{V}_{50}$ in Double-arc was lower than that of IMRT $(\mathrm{p}<0.05)$, while there was no statistical difference between Single-arc and IMRT. 


\section{Heart}

VMAT plans were superior in sparing the heart in terms of $\mathrm{D}_{\text {mean }}$ and $\mathrm{D}_{35 \%}$. The mean dose to heart for VMAT plans was better than IMRT plans by a marginal percentage variation $(1 \%-2 \%)$. Similar results were for $\mathrm{D}_{35 \%}$ heart doses. However, there was no statistical significance between these four plans, except that Single-arc plan showed a significant difference in heart mean dose when compared with 7f-IMRT plan. Details were in Table 4.

\section{Monitor Units and Delivery Time}

As displayed in Table 5, compared with IMRT plan, the total MU was reduced by $42.1 \%$ in VMAT plan. For single-arc plans, the value was $49.6 \%$, and $34.6 \%$ for double-arc plans. The average MU needed to deliver the dose of $60 \mathrm{~Gy}$ for Single-arc ( $423.5 \pm 52.1 \mathrm{MU})$ was found to be the least. Similarly, the average MU for the 5f-IMRT, 7f-IMRT and Double-arc were 868.2 $\pm 182.0 \mathrm{MU}, 870.0 \pm$ 225.3 MU and 548.8 $\pm 47.2 \mathrm{MU}$, respectively. The delivery time in VMAT plans reduced from 193.8 seconds to 99.2 second by around $48.8 \%$ compared to IMRT plans.

\section{Discussion}

Radiation technique develops from conventional radiotherapy to conformal radiotherapy, and then to IMRT. IMRT reduce the dose to lung parenchyma thus facilitating tumor dose escalation, which might improve local control and improves the uniformity of tumor irradiation and reduces the dose to lung. However, the disadvantage of IMRT is the higher number of MU and longer treatment time. The relative long treatment time will increase patients' discomfort. The prolongation of the fraction time will spare tumors with a fast DNA healing result in inefficient therapeutic benefits. And longer treatment times increase the likelihood of patient movement during treatment, which can potentially lead to PTV miss. VMAT, first raised by $\mathrm{Yu}$ in 1995 [14], was capable for intensity-modulated radiation delivery during gantry rotation with dynamic multi-leaf collimator (MLC) motion, variable dose rates (DR) and gantry speed modulation. Previous studies [4]-[9] showed VMAT can generate similar or better dose distributions including target volumes and OARs and achieve a reduction in treatment time and a reduction in monitor units (MU). Cervical-thoracic esophageal cancers because of its anatomically adjacent to spinal cord lead it hard to generate satisfactory dose distributions with spinal cord limits. This study compares the dosimetric parameters and radiobiological effects in cervical-thoracic esophageal cancers of four plans. The results indicated that VMAT could generate better radiotherapeutic plans than IMRT.

Concerning PTV, RA techniques (Single-arc: CI $=72.5 \pm 4.6$; Double-arc: CI $=72.1 \pm 5.1$ ) achieved slightly better conformality than IMRT (5f-IMRT: CI $=$ $70.4 \pm 7.1$; 7f-IMRT: $\mathrm{CI}=71.7 \pm 8.6$ ), whereas it resulted to be inferior on target coverage and dose homogeneity in comparison to IMRT. Both VMAT and IMRT plans resulted in almost equivalent $\mathrm{D}_{\text {mean }}$ of PTV. Though IMRT achieved better 
target coverage, it compromised over-irradiation of target volume. $\mathrm{V}_{107}$ was comparable $1.9 \%\left(\mathrm{CI}_{95 \%}:[0.7 \%, 3.4 \%]\right)$ for IMRT and VMAT.

Notwithstanding the considerable differences in target size ( $60 \%$ in the PTV), the plans for all techniques resulted in robust dosimetric parameters for the targets (as presented by the small standard deviations), manifesting that the analysis is robust against inter-patient variability.

Compared with IMRT, one of major advantage of VMAT was sparing OARs. Our study suggested Double-arc plans achieved the best of all plans in sparing cord. Double-arc plans spared 1.2 Gy on average when compared to IMRT plans. Although all plans fulfilled the limits of cord $\left(D_{\max }<45 \mathrm{~Gy}\right)$, more cord sparing could be easier to design better plans without compromised any OARs and target volumes. Another OAR was heart, VMAT reduced 0.3 Gy and 0.4 Gy on average on $\mathrm{D}_{\text {mean }}$ and $\mathrm{D}_{35 \%}$, respectively. Radiation-associated cardiac diseases were slowly progressing until 10 - 20 years after radiation. Although it was of minor importance for our comparison, the cardiac dose may become more important as cure rate improves, it was still desirable to reduce cardiac dose as much as possible.

The analysis of lung data showed that VMAT improved sparing compared to IMRT (Table 3). MLD and $\mathrm{V}_{20}$ as the two main predictors for lung toxicity were accounted. $\mathrm{V}_{20}$ was reduced by $3.2 \%$ on VMAT compared to IMRT. Similar results were also observed in MLD, where VMAT reduced MLD by $4.8 \%$ compared to IMRT. Emerging data [15] [16] suggested that percentage of lung volume receiving lower dose maybe a more excellent predictor of pulmonary toxicity, however this study demonstrated no statistic differences between VMAT and IMRT on $\mathrm{V}_{5}$.

VMAT was performed simultaneously with rotation by a dynamic MLC adaptation to the target volume during the rotation thus reduced the number of required MU. In this study, VMAT technique offered a clear benefit in terms of reduction in MU by an average $42.1 \%$ when compared with IMRT. The reduction on MU in turn leaded to reduce treatment delivery time from 193.8 to 99.2 seconds. Theoretically, the significant reduction of MU by VMAT decreases scattered dose and may reduce the risk of secondary malignancies. Radiation therapy treatments for the esophagus require the patient to be positioned supine with their arms extended above their head and clear of the treatment fields. This position can be very uncomfortable for many patients and the shorter treatment time afforded by VMAT could save patients a substantial amount of discomfort. And shorter treatment times reduce the likelihood of patient movement during treatment, which can potentially lead to PTV miss. In addition, shorter treatment times improved the utilization of machine.

\section{Conclusion}

For the treatment of cervical-thoracic esophageal cancer, VMAT is capable of delivering plans with better OAR sparing and without compromising PTV pa- 
rameters. In addition, VMAT technique can significantly reduce the number of monitor units required and overall treatment time. In a word, the VMAT technology can be an alternative method for cervical-thoracic esophageal cancer.

\section{Funding}

The study was partially supported by a grant from Guangdong Province innovation school project No. 2018KQNCX128, Xiamen Medical and Health Guidance Project No. 3502Z20209111.

\section{Conflicts of Interest}

The authors declare no conflicts of interest regarding the publication of this paper.

\section{References}

[1] Herskovic, A., Martz, K., al-Sarraf, M., Leichman, L., Brindle, J., Vaitkevicius, V., Cooper, J., Byhardt, R., Davis, L. and Emami, B. (1992) Combined Chemotherapy and Radiotherapy Compared with Radiotherapy Alone in Patients with Cancer of the Esophagus. The New England Journal of Medicine, 326, 1593-1598. https://doi.org/10.1056/NEJM199206113262403

[2] Minsky, B.D., Pajak, T.F., Ginsberg, R.J., Pisansky, T.M., Martenson, J., Komaki, R., Okawara, G., Rosenthal, S.A. and Kelsen, D.P. (2002) INT 0123 (Radiation Therapy Oncology Group 94-05) Phase III Trial of Combined-Modality Therapy for Esophageal Cancer: High-Dose Versus Standard-Dose Radiation Therapy. Journal of Clinical Oncology, 20, 1167-1174. https://doi.org/10.1200/JCO.2002.20.5.1167

[3] Xu, C., Xi, M., Komaki, R., Balter, P.A., Huang, M.L., Hobbs, B.P., Wang, L.H. and Lin, S.H. (2017) Dosimetric and Clinical Outcomes after Volumetric Modulated Arc Therapy for Carcinoma of the Thoracic Esophagus. Advances in Radiation Oncology, 2, 325-332. https://doi.org/10.1016/j.adro.2017.03.006

[4] Munch, S., Oechsner, M., Combs, S.E. and Habermeh, D. (2017) DVH-and NTCPBased Dosimetric Comparison of Different Longitudinal Margins for VMAT-IMRT of Esophageal Cancer. Radiation Oncology, 12, Article No. 128128. https://doi.org/10.1186/s13014-017-0871-3

[5] Huang, J.-H., Wu, X.-X., Lin, X., et al. (2019) Evaluation of Fixed-Jaw IMRT and Tangential Partial-VMAT Radiotherapy Plans for Synchronous Bilateral Breast Cancer Irradiation Based on a Dosimetric Study. Journal of Applied Clinical Medical Physics, 20, 31-41. https://doi.org/10.1002/acm2.12688

[6] Li, Y., Wang, J., Tan, L., Hui, B.N., Ma, X.W., Yan, Y.L., Xue, C.F., Shi, X.T., Drokow, E.K. and Ren, J. (2018) Dosimetric Comparison between IMRT and VMAT in Irradiation for Peripheral and Central Lung Cancer. Oncology Letters, 15, 3735-3745. https://doi.org/10.3892/ol.2018.7732

[7] Liu, Y.-C., Chang, H.-M., Lin, H.-H., Lu, C.-C. and Lai, L.-H. (2020) Dosimetric Comparison of Intensity-Modulated Radiotherapy Volumetric Modulated Arc Therapy and Hybrid Three-Dimensional Conformal Radiotherapy/Intensity-Modulated Radiotherapy Techniques for Right Breast Cancer. Journal of Clinical Medicine, 9 , 3884. https://doi.org/10.3390/jcm9123884

[8] Bedford, J.L., Hansen, N.V., McNair, H.A., Aitken, A.H., Brock, J.E., Warrington, A.P. and Brada, M. (2008) Treatment of Lung Cancer Using Volumetric Modulated 
Arc Therapy and Image Guidance: A Case Study. Acta Oncologica, 47, 1438-1443. https://doi.org/10.1080/02841860802282778

[9] Bertelsen, A., Hansen, O. and Brink, C. (2012) Does VMAT for Treatment of NSCLC Patients Increase the Risk of Pneumonitis Compared to IMRT? - A Planning Study. Acta Oncologica, 51, 752-758. https://doi.org/10.3109/0284186X.2011.648341

[10] Gibbons, J.P. and Khan, F.M. (2014) The Physics of Radiation Therapy. Lippincott Williams \& Wilkins, 20, 439.

[11] Sriram, P., Syamkumar, S.A., Kumar, J.S., Prabakar, S., Dhanabalan, R. and Vivekanandan, N. (2012) Adaptive Volumetric Modulated Arc Treatment Planning for Esophageal Cancers Using Cone Beam Computed Tomography. Physica Medica, 28, 327-332. https://doi.org/10.1016/j.ejmp.2011.10.006

[12] Van Benthuysen, L., Hales, L. and Podgorsak, M.B. (2011) Volumetric Modulated Arc Therapy vs. IMRT for the Treatment of Distal Esophageal Cancer. Medical Dosimetry, 36, 404-409. https://doi.org/10.1016/j.meddos.2010.09.009

[13] Yin, Y., Chen, J., Xing, L., Dong, X., Liu, T., Lu, J. and Yu, J. (2011) Applications of IMAT in Cervical Esophageal Cancer Radiotherapy: A Comparison with Fixed-Field IMRT in Dosimetry and Implementation. Journal of Applied Clinical Medical Physics, 12, 48-57. https://doi.org/10.1120/jacmp.v12i2.3343

[14] Yu, C.X. (1995) Intensity-Modulated Arc Therapy with Dynamic Multileaf Collimation: An Alternative to Tomotherapy. Physics in Medicine \& Biology, 40, 1435-1449. https://doi.org/10.1088/0031-9155/40/9/004

[15] Vogelius, I.S., Westerly, D.C., Cannon, G.M., Mackie, T.R., Mehta, M.P., Sugie, C. and Bentzen, S.M. (2011) Intensity-Modulated Radiotherapy Might Increase Pneumonitis Risk Relative to Three-Dimensional Conformal Radiotherapy in Patients Receiving Combined Chemotherapy and Radiotherapy: A Modeling Study of Dose Dumping. International Journal of Radiation Oncology Biology Physics, 80, 893-899. https://doi.org/10.1016/j.ijrobp.2010.12.073

[16] Vogelius, I.R., Westerly, D.C., Aznar, M.C., Cannon, G.M., Korreman, S.S., Mackie, T.R., Mehta, M.P. and Bentzen, S.M. (2011) Estimated Radiation Pneumonitis Risk after Photon Versus Proton Therapy Alone or Combined with Chemotherapy for Lung Cancer. Acta Oncologica, 50, 772-776.

https://doi.org/10.3109/0284186X.2011.582519 